Ricardo Granero 1

Reinaldo Ortiz 1

Orlando Gómez-M arín 2

César I saacura 1

Al exis Goyo 1

José Rodriguez 1

\section{Validación de "infarto de miocardio" como causa de muerte en certificados de defunción, Barquisimeto, Estado Lara, Venezuela}

\author{
Validation of "myocardial infarction" as cause \\ of death on death certificates in Barquisimeto, \\ Lara state, Venezuela
}

1 Centro Cardiovascular Regional Centro Occidental ASCARDIO. Apartado de Correos 495. Barquisimeto, 3002. Estado Lara, Venezuela. 2 Departments of Epidemiology and Public Health and Pediatrics, University of Miami School of Medicine. Dominion Tower, P.O. Box 016069, Miami, Florida, 33101, USA.
Abstract Death rates due to Ischemic Heart Di sease (IHD) are widely used to assess regional and national cardi ovascular health status. However, their validity is questionable, essentially because they are based on death certificates (DCs). The present study was conducted in the Greater Metropolitan Area of Barquisi meto (Lara State, Venezuela), from June 1991 to July 1992. The purpose was to establish the validity of the diagnosis of myocardial infarction in DCs of intrahospital deaths. Efforts were made to locate all clinical charts and any other relevant information. To validate the diagnosis of MI in the DC we used the MONICA project criteria. During the study period a total of 247 eli gible DCs were recorded. Sufficient clinical information was located for 136 cases (55\%). The results were as follows: definite MI - 62 cases (45.6\%); probable MI - 31 cases (22.8\%); and non $\mathrm{MI}-43$ cases (31.6\%). These results may indicatean overestimation of MI death rates of about $32 \%$ [95\% Confidence Interval $=23.8 \%-39.4 \%]$. Since these data arefrom a region with an organized cardi ovascular health system, it is expected that the overestimation for the rest of the country may be even higher. Thus, in order to enhance quality and accuracy of DCs, it is necessary to devel op a system to improveadherence to existing norms for filling out and processing such forms. Key words Myocardial Infarction; Validity; Death Certificate; Epidemiology

Resumen Las tasas de mortalidad por cardiopatía isquémica $(\mathrm{Cl})$ han si do utilizadas ampliamente en el diagnóstico de la salud cardiovascular de regiones y países. Sin embargo, su validez ha sido cuestionada fundamentalmente por lo quele da origen: el certificado de defunción (CDD). El presente estudio tiene el objetivo de establecer la vali dez del diagnóstico “infarto de mi ocardio (IM)" como causa de muerte en Ios CDD de muertes intra-hospitalarias en la ciudad de Barquisimeto, Estado Lara, Venezuela. Entrejulio de 1991 y junio de 1992, se identificaron todos los CDD con las característi cas mencionadas, se trató de ubicar la historia clínica correspondiente a cada caso y cualquier otra información clínica pertinente. Se registraron 247 CDD el egi bles y se ubicó información clínica adecuada en 136 casos (55\%). Aplicando los criterios del Proyecto MONICA, se encontraron M-definitivo en 62 casos (45,6\%), I M-probable en 31 casos (22,8\%) y No-IM en 43 casos (31,6\%). Los resultados indican que existe un sobre registro de casos de muerte por IM del 32\% (interval o de confianza del $95 \%=23,8-39,4$ ). A la luz de estos resultados es necesario estudiar los factores que inciden en la exactitud de los diagnósticos I M en I os CDD. Con esto tendríamos elementos para revisar las normas y los procedimientos empleados en la producción y manejo de Ios CDD, así como preparar un programa de educación para mejorar la precisión delos mismos. Palabras clave Infarto de Miocardio; Validación; Certificado de Defunción; Epidemiología 


\section{Introducción}

Estudios epidemiológicos sobre mortalidad son necesarios en el análisis de la salud de los pueblos. Estos aportan información imprescindible en el proceso de decisión sobre políticas de salud, y por ende, en el establecimiento de programas preventivos y curativos. Las tasas de mortalidad constituyen el indicador fundamental de la medida de mortalidad, y por eso son ampliamente usadas para describir y comparar el estado de salud de regiones y países. Esas comparaciones han permitido evidenciar que existen similitudes y diferencias que, en muchos casos, han constituído la base para elaborar teorías sobre las causas y los factores de riesgo contribuyentes a la ocurrencia de determinadas entidades nosológicas. En el caso específico del infarto de miocardio (IM), la disparidad en las tasas de mortalidad en diferentes países ha permitido hipotizar sobre su causalidad y los factores de riesgo que contribuyen a su surgimiento. Ejemplos de estos son, hipertensión arterial, actividad física, hábito tabáquico, obesidad y alimentación, entre otros. En algunos casos la variación de las tasas ha sido presentada como indicativo de la efectividad de programas preventivos (Marmot, 1992, 1985; Stamler, 1989; Wilhelmsen et al., 1989; Thomson et al., 1992). En el caso del IM se plantea que las tasas de mortalidad son un reflejo de las tasas de morbilidad. Esta aseveración es lógica y biológicamente razonable. Sin embargo, ha sido cuestionada desde diferentes puntos de vista, uno de ellos es la validez de los datos que le da origen: el certificado de defunción (CDD) (Folsom et al., 1987; Lee et al., 1990). El proceso de validación es complicado, y presenta dificultades tales como difícil acceso a las fuentes de datos, baja prevalencia de autopsia, y registros con pocos datos clínicos relevantes. Datos del estudio MONICA indican que la validez de los CDD varía de acuerdo al país estudiado (Tunstall, 1994; Tuomilehto, 1992). En Venezuela, las enfermedades cardiovasculares ocupan el primer lugar en la mortalidad de personas mayores de 35 años. Hasta donde sabemos no existen datos publicados que permitan tener una idea sobre la validez de los CDD en el área metropolitana de Barquisimeto. Por lo tanto no es posible conocer el grado de exactitud de las tasas de mortalidad.

\section{Materiales y métodos}

El Ministerio de Sanidad y Asistencia Social (MSAS) es el organismo encargado de recolectar los CDD de las muertes que ocurren en la región. Entre Julio 01 de 1991 y Junio 30 de 1992, personal entrenado revisó semanal mente todos los CDD de la oficina de epidemiología del Sub-Sistema de Salud del Estado Lara. Se seleccionaban todos CDD correspondientes a muertes ocurridas en el área metropolitana de Barquisimeto, que establecían en forma literal infarto de miocardio (I M) como causa de muerte. Barquisimeto está localizada en el Estado Lara, Venezuela con una población, en el momento del estudio, de un millón de habitantes. En el presente artículo solamente se presentan los resultados correspondientes a muertes intra-hospitalarias, ya sea muertes ocurridas dentro de un hospital, centro de salud público o privado.

A medida que se seleccionaban los CDD, se procedió a extraer la información necesaria para el proceso de validación. Dentro del mes siguiente a la recepción del CDD, con previo consentimiento de las gerencias de los respectivos centros clínicos, se procedió a la busqueda de las historias clínicas. Se llevó a cabo toda diligencia razonable para localizar las historias clínicas u otra información relevante para el proceso de validación. En este sentido, se trató de ubicar información en centros de salud incluyendo el Centro Cardiovascular Regional Centro Occidental (ASCARDIO). ASCARDIO es una organización no gubernamental (ONG) proveedora de servicios clínicos en el área de salud cardiovascular. Esta ONG presta atención a una proporción muy alta de la población local.

Personal previamente entrenado, residentes en Cardiología, extraía la información contenida en la historia clínica utilizando un formato basado en los criterios para IM del proyecto MONICA, a saber: síntomas, hallazgos de ECG, enzimas y necropsia (WHO, 1990). La Tabla 1 muestra un resumen de los criterios según su categoría. De acuerdo al Manual de MONICA y en base a los datos obtenidos de la historia clínica, se clasificaron los casos como: IM definitivo, IM o muerte coronaria posible, No IM (WHO, 1990).

Los datos recolectados en los formularios fueron transcritos a un archivo de computadora y analizados con el paquete estadístico EpiInfo version 6.0. Para asegurar el anonimato se omitieron los nombres de los difuntos y de los centros de salud a los cuales les fue asignado un código. 
Tabla 1

Criterio para clasificación de infarto de miocardio.

I. Infarto de miocardio definitivo

a) Electrocardiograma (ECG) definitivo ${ }^{1}$.

b) Síntomas típicos, atípicos o descritos inadecuadamente, junto con un ECG probable y enzimas anormales².

c) Síntomas típicos y enzimas anormales con ECG isquémico, no codificable o ECG no disponible.

d) Muerte, súbita o no, donde, en la necropsia, la inspección macroscópica sea compatible con infarto de miocardio y/o una oclusión coronaria reciente.

II. Infarto de miocardio posible

Muerte, súbita o no, donde no hay una buena evidencia de otra causa de muerte, clínica o en la necropsia.

a) Con síntomas típicos, atípicos o inadecuadamente descritos.

b) Sin síntomas típicos, atípicos o inadecuadamente descritos, pero con evidencia de una oclusión coronaria crónica, estenosis o una cicatriz en el miocardio en la necropsia.

c) Con una buena historia de enfermedad coronaria crónica tal como infarto de miocardio definitivo, posible, insuficiencia coronaria, angina pectoris en ausencia de una enfermedad valvular importante o de cardiomiopatía.

III. No infarto de miocardio

Muerte, súbita o no, no en la categoría I, donde otro diagnóstico ha sido planteado.

1 y 2: Criterios para ECG y enzimas (Tunstall et al., 1994; WHO, 1990).

\section{Resultados}

En el período comprendido entre Julio 01 de 1991 y Junio 30 de 1992, 367 CDD con infarto de miocardio como causa más probable de muerte, se registraron en el área metropolitana de la ciudad de Barquisimeto. La distribución de los casos, según el lugar de la muerte de acuerdo al CDD, es de: muertes hospitalarias $247(67,3 \%)$ y muertes extra-hospitalarias 120 (32,7\%). De los 247 casos intra-hospitalarios, se localizó la historia clínica completa con datos relevantes en 96 casos, para un 38\% del universo en estudio (247 casos). En 40 casos adicionales, con historia clínica incompleta, se identificó información relevante en otras fuentes. Por lo tanto, 136 casos (55\%) fueron incluídos en el análisis final. El estudio de estos 136 casos indicó que el $58,4 \%$ correspondía a hombres, y que la edad promedio (intervalo de confianza del 95\%) fue de 65 (62-66) años para los hombres y 68 (64-69) para las mujeres. La distribución de la especialidad del médico firmante del CDD fue: médico general o residente, $46,4 \%$; patólogo, 35,4\%; internista, 7,1\%; cardiólogo, $4 \%$; y el $7,1 \%$ restante no codificable. Solamente se realizó necropsia en 23 (17\%) de los casos admitidos al estudio, de los cuales en 4 casos los datos fueron insuficientes o no fue posible localizar el protocolo del procedimiento. El grupo de casos donde no fue posible encontrar información, 111 casos, no diferió significativamente del grupo incluído en el estudio en cuanto a edad, sexo, lugar de de- función o categoría del médico firmante del CDD.

LaTabla 2 presenta un resumen de la composición de los casos incluídos de acuerdo al origen de los datos utilizados para la validación, estos fueron: por historia clínica en el lugar de la muerte en $55,6 \%$, por los hallazgos de la necropsia en $14,7 \%$, y por historia clínica incompleta más antecedentes en la historia de ASCARDIO en $29,7 \%$ de los casos.

Utilizando los criterios mencionados se encontró, I M-definitivo en 62 casos (45,6\%); IMprobable en 31 casos $(22,8 \%$ ) y No-IM en 43 casos $(31,6 \%)$. Los resultados indican que en la región mencionada existe un sobre registro de la mortalidad por IM del 31,6\% (intervalo de confianza del $95 \%=23,8 \%-39,4 \%$ ).

\section{Discusión}

La singularidad del presente trabajo consiste en que la selección de los CDD se realizó en base a lo escrito en el CDD original. En otros estudios la selección se realizó retrospectivamente mediante códigos de la clasificación internacional en un banco de datos (Folsom et al., 1987; Lee et al., 1990). De tal manera que, en este trabajo se valida el diagnóstico literal "infarto de miocardio" que el médico asignó al caso y no la interpretación del nosologista. Según los datos de CDD, el 67,3\% (247) de todos los IM se produjeron en centros asistenciales. La proporción de muertes por IM fuera del hospi- 
Tabla 2

Distribución de casos de acuerdo al origen de los datos para validación.

\begin{tabular}{lrc}
\hline Tipo de casos & $\mathrm{n}$ & $\%$ \\
\hline Historia clínica con datos suficientes & 77 & 55,6 \\
Historia clínica y necropsia & 19 & 14,7 \\
Historia clínica insuficiente más historia en ASCARDIO & 40 & 29,7 \\
Total & 136 & 100 \\
\hline
\end{tabular}

ASCARDIO : Centro Cardiovascular Centro Occidental

tal varía según las poblaciones estudiadas. En Minnesota (EEUU), es de 40\% (Folson et al., 1987); en Carolina del Norte (EEUU), la proporción oscila entre 39 y $45 \%$ dependiendo del sexo y de la raza (Lee et al., 1990).

En nuestro estudio, 136 casos (55\%) fueron incluídos en el análisis final. Esta cifra es menor que la reportada por la mayoría de los centros participantes en el Proyecto MONICA, donde en 38 poblaciones el promedio de muertes no clasificables fue del $22 \%$, en 2 poblaciones $55 \%$ y en 15 poblaciones el 33\% (Tunstall et al., 1994). En un estudio colaborativo en los EEUU, no fue posible localizar resultados sobre enzimas y ECG en un total de $35 \%$ de hombres blancos, $40 \%$ de mujeres negras, y $30 \%$ de mujeres blancas (Lee et al., 1990). La proporción de casos no incluídos (45\%) debe ser tomada en cuenta al interpretar los resultados. En este sentido, se podría decir que el grupo incluído parece ser representativo del universo a estudiar. Esta afirmación se basa en el hecho de que ambos grupos no difieren, significativamente, en la distribución de importantes variables como sexo, edad, lugar de defunción o categoría del médico firmante del CDD. La disponibilidad de datos clínicos completos parece depender de varios factores como la raza, el sexo del paciente y el nivel socio-económico (Lee et al., 1990). En nuestro estudio, la recolección de los datos resultó ser una tarea complicada y laboriosa. El desorden administrativo fue el problema logístico más común. La disparidad de los datos plasmados en el CDD con respecto a la realidad, fue el segundo problema en importancia que impidió localizar la historia clínica en el centro donde supuestamente se produjo la defunción. Estos son problemas anticipables en todo estudio retrospectivo. Como uno de nuestros objetivos, la metodología empleada en este estudio asegura la comparabilidad con los resultados de MONICA.

El 31,6\% (IC95\% =23,8\%-39,4\%) de los casos estudiados no correspondía a IM. Esta cifra es mayor que la encontrada en los centros par- ticipantes en MONICA. Por ejemplo, en Finlandia (FINMONICA), la mortalidad coincidió con los registros oficiales de mortalidad (Tuomilehto, et al., 1992); en Lombardía, los diagnósticos coronarios reportados en Ios CDD en la década de 1980 tienen una sensibilidad del 87\% y un Kappa del 0,35 (Cesana et al., 1992); en Nueva Zelandia y Australia, los resultados de MONICA se correlacionan favorablemente con las tasas oficiales; todo lo cual indica la precisión de los CDD (Cesana et al., 1992; Beaglehole et al., 1989). Estos resultados provienen de países selecionados en forma sesgada, con sistemas de registros e infraestructura más adecuada para lograr la recolección de los datos (Tunstall et al., 1994). Sin embargo, hay evidencias de variabilidad en la exactitud del CDD dentro de un mismo país. En EEUU, un estudio reportó que el diagnóstico IM era menos específico para negros que para blancos, y concluye que la alta tasa de fatalidad del IM en negros, así como la el evada tasa de diagnóstico erróneo (o su codificación), pueden explicar la diferencia en las tasas de mortalidad por enfermedad coronaria entre blancos y negros (Lee et al., 1990). En este estudio, no intentamos evaluar aquellos casos de muertes por IM cuyo CDD indica otra causa de muerte. Por esto no disponemos de datos para evaluar el sub-registro, lo que sería motivo de un estudio diferente a fin de complementar este trabajo.

En 1992, las tasas de mortalidad en Venezuela, por códigos 410-414 (CIE Novena edición), fueron para varones de 108, hembras 81 y un total de 95 (tasas por 100.000 habitantes y estandarizadas utilizando la población del nuevo mundo según la OMS). Si se considera el sobre registro del $31,6 \%$ y se asume que es posible extrapolarlo a Venezuela, las tasas serían para varones de 76, hembras 54 y un total de 64 por 100.000 habitantes. Lo que representa 3864 muertes menos que las oficialmente reportadas por IM en Venezuela en 1992.

A la luz de estos resultados, es necesario estudiar más profundamente los factores que inciden en la exactitud de los diagnósticos IM en Ios CDD, con lo que tendríamos elementos adecuados para revisar las normas y los procedimientos empleados en la producción y manejo de los CDD, así como preparar un programa de educación para mejorar la precisión de los mismos. 


\section{Referencias}

BEAGLEHOLE, R.; DOBSON, A.; HOBBS, M.; JACKSON, R. \& MARTIN, C., 1989. Cardiovascular disease in Australia and New Zeland. International Journal of Epidemiology, 18:145-148.

CESANA, G.; FERRARIO, M.; SEGA, R.; BRAVI, R.; GUSSONI, M.; DE VITO, G. \& VALAGUSSA, F., 1992. Drop in cardiovascular and coronary mortality in Lombardia, 1969-1987. Giornale Italiano di Cardiologia, 22:293-305.

FOLSOM. A.; GÓMEZ-MARÍN, O.; GILLUM, R.; LOHMAN, W. \& JACOBS, D., 1987. Out-of-hospital coronary death in an urban population-validation of death certificate diagnosis. American Journal of Epidemiology, 125:1012-1018.

JACKSON, R.; STEWART, A. \& BEAGLEHOLE, R., 1990. Trends in coronary heart disease mortality and morbidity in Auckland, New Zealand, 1974-1986. International Journal of Epidemiology, 19:279283.

LEE, M.; BORHANI, N. \& KULLER, L., 1990. Validation of reported myocardial infarction mortality in blacks and wites. A report from the community cardiovascular surveillance program. Annals of Epidemiology, 1:1-12.

MARM OT, M., 1992. Coronary heart disease: rise and fall of a modern epidemic. In: Coronary Heart Disease Epidemiology (M. Marmot \& P. Elliot, eds.), pp. 3-19, New York: Oxford University Press.

MARM OT, M. 1985. Interpretation of trends in coronary heart disease mortality. Acta Medica Scandinavica, 701:58-65.
STAMLER, J., 1989. Opportunities and pitfalls in international comparisons related to patterns, trend and determinants of CHD mortality. International Journal of Epidemiology, 18:S3-18.

THOMSON, P.; PARSONS, R.; JAM ROZIK, K.; HOCKEY, R.; HOBBS, M. \& BROADHURST, R., 1992. Changing patterns on medical treatment in acute myocardial infarction. Observations from the MONICA Project 1984-1990. Medical Journal of Australia, 20:87-92.

TUNSTALL, H.; KUULASM AA, K.; AMOUYCL, P.; ARVEILER, D.; RAJAKANGAS, A. \& PAJAK, A., 1994. Myocardial infarction and coronary death in the World Health Organization MONICA Project. Registration procedures, event rates and case-fatality rates in 38 populations from 21 countries in four continents. Circulation, 90:583-612.

TUOMILEHTO, J.; ARSTILA, M.; KAARSALO, E.; KANKAANPAA, J.; KETONEN, M.; KUULASM AA, K.; LEHTO, S.; MIETTINEN, H.; MUSTANIEMI, H. \& PALOMAKI, P., 1992. Acute myocardial infarction in Finland: baseline data from the FINMONICA AMI register in 1983-1985. European Heart Journal, 13:577-587.

WILHELMSEN, L.; JOHANSSON, S.; ULVENSTAM, G.; WELIN, L.; ROSENGREN, A.; ERIKSSON, H.; LARSSON, B.; TSIPOGIANNI, A. \& WEDEL, H., 1989. CHD in Sweden: mortality, incidence and risk factors over 20 years in Gothenburg. International Journal of Epidemiology, 18 (Suppl. 1):S101-108.

WHO (World Health Organization), 1990. Monica Manual, Revised Edition. Geneva: WHO. MONICA Project, Cardiovascular Disease Unit. 